\title{
LOS METATAGS EN EL COMERCIO ELECTRÓNICO. UN ANÁLISIS DE LA JURISPRUDENCIA NORTEAMERICANA Y LA DOCTRINA ESPAÑOLA
}

\author{
Carlos Amunátegui Perelló*
}

\begin{abstract}
RESUMEN: Este artículo trata de los problemas de marcas y competencia desleal que genera el uso de metatags en las páginas Web en la jurisprudencia norteamericana y su posible aplicación en el sistema jurídico español.
\end{abstract}

Palabras clave: Metatag, marcas, competencia desleal.

ABSTRACT: This article discusses the problems that the use of metatags in Internet pages generates in North American jurisprudence and the application of this principles in the Spanish law system.

Key words: Metatag, trademarks, cutthroat competition.

\section{INTRODUCCIÓN}

El comercio electrónico, durante el transcurso de su breve existencia, ha planteado numerosos problemas jurídicos en su expansión. Por lo común, tales situaciones tienden a generarse primeramente en los Estados Unidos por el alto nivel de desarrollo comparativo de que goza Internet en dicho país, para después convertirse en problemas comunes para el resto de la comunidad internacional. Tal situación ha provocado un aumento explosivo en la importancia de su jurisprudencia, la que suele convertirse en un punto de apoyo para el resto de los países a la hora de evaluar nuevas soluciones a fin de enfrentar los desafíos que esta forma de intercambio plantea.

Así como los conflictos entre nombres de dominio y marcas fueron solucionados con una relativa eficacia usando los conceptos desarrollados por los tribunales norteamericanos, actualmente se producen nuevos focos de interés hacia otras áreas de su jurisprudencia, desde donde pueden preverse casos y soluciones posibles para el futuro.

Uno de los temas más controvertidos actualmente en el medio norteamericano, y que sin embargo tiene aún una escasa recepción en Europa, es el tema de los metatags. A través de ellos, situaciones de una creciente complejidad se han ido paulatinamente suscitando en los Estados Unidos, donde problemas relacionados con Derecho de Marcas y Competencia Desleal se han manifestado en repetidas oportunidades a contar del mes de septiembre de 1997. No obstante que tales conflictos no se reflejan aún en la jurisprudencia española, es fácil observar que su ocurrencia es predecible para los próximos años y de ahí el interés doctrinario y práctico del presente trabajo.

\footnotetext{
* Profesor de Derecho Romano, Pontificia Universidad Católica de Chile.
} 
El método que utilizaremos al analizar el problema es sencillo. En primer término daremos un concepto que nos permita comprender la función e importancia de los metatags, para luego comenzar a estudiar las cuestiones jurídicas que han suscitado, primeramente a través de la jurisprudencia norteamericana, para luego pasar a estudiar las posibles consecuencias que tal comportamiento tendría en el Derecho Español.

\section{2. ¿QUÉ SON LOS METATAGS?}

Las páginas que componen la World Wide Web (Red en adelante) tienen una doble cara. Por una parte está aquel conjunto de fotos, sonidos y textos que aparecen proyectadas en el ordenador del usuario de Internet al acceder a ella, y por otra está su Código Fuente, que corresponde a la programación misma que permite al computador interpretar como un hipertexto la información a la que accede a través de la Red. Dicho Código Fuente está escrito en HTML (Hyper Text Markup Language), que consiste en una cantidad de órdenes o tags construidos en un lenguaje sencillo que normalmente aparece oculto a la vista del usuario, salvo que decida visualizarlo mediante la opción adecuada de su navegador.

De esta manera, mientras el usuario "ve" en su pantalla un determinado conjunto de información, el verdadero contenido de la página permanece escondido y es "comprendido" solo por el ordenador a fin de configurar la información recibida como un hipertexto.

Los tags regulares constituyen información relativa a la disposición y despliegue de la página en pantalla, como su color, el tipo de caracteres, las fotografías que aparecerán, su ubicación, etc. Junto a estos tags existen otros que no tienen por finalidad manifestarse en la pantalla del usuario, sino que contienen información que permanece oculta a este, pero que es manifiesta para otros ordenadores. Estos son los metatags, que no obstante ser invisibles para el usuario suelen reunir las palabras clave de la información más relevante en la página visitada.

La finalidad de los metatags se encuentra estrechamente vinculada con los "buscadores" de páginas en la Red. Cuando el usuario desea ubicar información sobre un tema determinado introduce en su buscador (sea Lycos, Yahoo, Altavista u otro similar) ciertas palabras o frases relacionadas con el asunto en cuestión. En este momento el buscador da una mirada a los metatags de las páginas de la Red a fin de encontrar qué lugares contienen entre ellos alguna designación relativa a la solicitada, formando de esta manera una lista que responda a las necesidades del usuario.

Los sitios que contengan en sus metatags mayor cantidad de palabras iguales a las solicitadas aparecerán en primer lugar de la lista elaborada en razón de la búsqueda; los que menos, en los puestos siguientes.

Así, los metatags actúan de manera similar a un faro dentro de la Red, atrayendo la atención de los buscadores hacia ellos a fin de señalar qué información contiene la página en cuestión. De tal manera, constituyen un elemento de alta importancia en la Red pues contribuyen a su organización interna haciéndola accesible a los usuarios y útil como medio de búsqueda de información. 
Según el uso de los metatags se popularizó por parte de los buscadores, se hizo evidente que dichos mensajes contenidos en la página podían tener también una finalidad publicitaria. Así, un sitio que contuviese los metatags más utilizados en la Red aparecería entre los resultados de la mayor parte de las búsquedas, por lo que sería un lugar comúnmente visitado por los navegantes. Puesto que el tráfico que tenga una página determina su valor comercial para efectos de introducir publicidad en ella, como también su poder para atraer potenciales clientes a sus tiendas virtuales, comenzó a ser un lugar común de las páginas de la Red el tener largas listas de metatags, muchas veces con palabras no relacionadas con su contenido, a fin de atraer a potenciales clientes hacia ellas. Uno de los casos más llamativos fue la tendencia de las páginas de pornografía al introducir la palabra MIR dentro de sus metatags, a fin de captar a quienes estuviesen buscando información relativa a la estación espacial rusa ${ }^{1}$.

Otra forma de utilizar tales metatags como publicidad consiste en introducir los nombres de páginas que ofrezcan servicios relacionados con la información solicitada. Un ejemplo clásico puede obtenerse introduciendo la palabra "Disney" en cualquier buscador, donde aparecerá, amén de la información solicitada, una larga lista de agencias de viajes que ofrecen sus servicios para llevar al interesado al destino turístico.

Si bien estas situaciones crean un cierto desorden en la Red, pues aumentan innecesariamente el tamaño de las listas de resultados obtenidas en los buscadores, no fue sino hasta que ciertas empresas decidieron incluir los nombres de sus competidores directos entre sus metatags que se generó un problema jurídico propiamente.

En efecto, a fin de aparecer en los primeros lugares de las búsquedas muchos diseñadores de páginas para la Red comenzaron a incluir entre sus metatags no solo las palabras clave que describían el servicio ofrecido por la página en cuestión, sino también la lista de sus competidores potenciales más relevantes a fin de atraer a los clientes de estos a sus propias páginas.

Tal situación creó una serie de conflictos jurídicos en los Estados Unidos de Norteamérica, que serán el objeto principal del presente trabajo, tanto por su importancia dentro del comercio electrónico como por la alta probabilidad que existe de que tales situaciones se repitan en el medio español, donde aún no existe jurisprudencia relevante en el tema.

\section{GRUPOS DE CASOS}

A fin de tratar adecuadamente el problema en cuestión, dividiremos la materia en tres grupos de casos que se han dado en el Derecho estadounidense, acompañando a tales puntos con una necesaria reflexión relativa al estado del Derecho español en la materia.

En primer término centraremos nuestra atención en el modelo inicial de casos, que se manifiesta como un típico problema de la contraposición entre marcas y domi-

\footnotetext{
${ }^{1}$ Más información en el artículo de JACKSON (1997).
} 
nios en la Red, siendo en ellos los metatags un elemento más a considerar. En estos los metatags son un indicio más que lleva al Tribunal a concluir que hay un riesgo de confusión entre la página del propietario del dominio y la empresa del titular de la marca. Seremos necesariamente breves en tal análisis, puesto que para la presente investigación no nos interesa más que una manera secundaria tal conflicto, al existir una amplia variedad de jurisprudencia y libros de texto que se refieren a tal asunto. Por otro lado, en tales casos el tema de los metatags suele ser tratado como un simple apéndice del conflicto de confusión en las marcas, por lo que su importancia es muchas veces pasada por alto.

Un segundo grupo de casos se relaciona más directamente con la competencia desleal, donde entran a jugar factores como la competencia parasitaria y el riesgo de confusión de manera independiente de la legislación relativa a las marcas. Este es el punto donde centraremos nuestra atención de manera preferente, al tratarse de un tema relativamente virgen y que reviste una importancia jurídica elevada.

Finalmente observaremos un grupo de casos en que el uso de metatags parece legítimo y no presenta temas sensibles en materias jurídicas, por lo que su uso parece justificado. Tal sección es de importancia por prestar utilidad a la hora de tomar una decisión relativa al diseño de una página para la Red.

3.1. Casos de confusión relativos a marcas. El tema de los metatags hizo su primera aparición en el mundo jurídico norteamericano en relación a esta clase de casos.

En efecto, la situación se produjo en razón de la sentencia que resuelve la pretensión de Playboy Enterprises, Inc (en adelante PEI) contra Calvin Designer Label (CDL) de fecha 8 de septiembre de 1997.

El problema se enmarcaba dentro de los clásicos supuestos de confusión de marcas comprendido por el Lanham Act norteamericano. En dicho caso CDL había creado dos páginas pornográficas cuyos dominios eran www.playboyxxx.com y www.playmatelive.com, incluyéndose en ellos las marcas registradas Playboy y Playmate de las cuales PEI es titular.

A mayor abundamiento, tales sitios daban la falsa apariencia de ser auspiciados por PEI, toda vez que incluían frases que inducían a pensar al usuario que dichos sitios pertenecían o estaban de alguna manera autorizados por tal empresa. Amén de ello, en los metatags de las páginas de CDL se encontraban numerosas referencias "playboy" y "playmate", lo que ocasionaba que los usuarios que intentasen encontrar el sitio en la Red que pertenecía a PEI a través de un buscador, rápidamente fuesen desviados hacia los sitios pertenecientes a CDL en la errónea creencia de haber logrado su objetivo.

Como era esperable, la cuestión fue rápidamente resuelta en favor de PEI ordenando a CDL que cancelara sus dominios en la Red que incluyesen las palabras "playboy" o "playmate", como también su eliminación de los correspondientes metatags.

La decisión se encuentra claramente justificada en razón del Lanham Act, que protege a las marcas registradas de todo acto que tenga por fin inducir a confusión al público respecto a la titularidad o procedencia de los productos ofrecidos. De esta manera establece en su Sección 43 como ilegítimo y protege al titular de una marca de: 
"any person who, on, or in connection with any goods or services... uses in commerce any word, term, name, symbol, or device, or any combination thereof, or any designation of origin, false or misleading description of fact, or false or misleading representation of a fact which:

(1) is likely to cause confusion, or to cause mistake, or to device as to the affiliation, connection or association of such person with another person, or as to the origin, sponsorship, or approval of his or her goods, services, or commercial activities by another person"...

En otras palabras, en este caso fue sancionado el intento ilegítimo de CDL de hacerse pasar como un asociado o patrocinado de PEI en sus páginas a través del uso de las marcas de PEI tanto en sus dominios como en sus metatags.

Otro caso similar tuvo lugar más recientemente entre Columbia Insurance Company y seescandy.com y otros ${ }^{2}$. En este caso, existía una compañía llamada See's Candy Shops, Inc (See's) que cedió sus intereses a Columbia Insurance Company. Dicha compañía tenía un sitio en la Red dedicado a la venta de dulces de alta calidad, cuyas respectivas marcas estaban registradas a su nombre. Paralelamente, un grupo de individuos y compañías ficticias demandadas obtuvieron los dominios de sitios en la Red cuyos nombres eran altamente similares a los de See's, usando a la vez metatags con la marca de la misma a fin de atraer a su potencial clientela.

Nuevamente el resultado fue contrario a los demandados puesto que su finalidad consistía en hacer pasar su empresa por aquella que era efectivamente propietaria de la marca registrada See's Candy, lo que se adaptaba al presupuesto clásico del acto de confusión dentro del Derecho de Marcas. Consecuentemente, los demanadados fueron condenados a retirar sus dominios de la Red y no incluir dentro de sus metatags las marcas de los demandantes.

Como puede observarse con facilidad, las situaciones antes descritas no tratan los metatags en su estado "puro", sino que más bien como elementos anexos relacionados con problemas de titularidad de dominios frente a marcas, donde los metatags son un elemento que ayuda a crear confusión y dilución de las marcas registradas. Son situaciones cuya ocurrencia era más frecuente en los primeros tiempos de la Red, cuando los lindes del derecho de marcas no estaban claramente diferenciados de los dominios, y como consecuencia, tienen hoy en día un interés limitado.

En el Derecho español tal situación se encuentra solucionada por el art. $7^{\circ}$ de la Orden de 21 de marzo de 2000 del Ministerio de Ciencia y Tecnología que exige que el registro de dominios de Internet se lleve en coordinación con los Registros Públicos, especialmente con la Oficina Española de Patentes y Marcas, lo que limita claramente la posibilidad de inscribir como dominio una marca registrada de conformidad a la ley. No obstante, aun en el caso de producirse, el titular de la marca puede accionar en contra del titular del dominio amparándose en el art. 31 de la LM que lo autoriza a dirigirse en contra de "los

${ }^{2}$ Columbia Insurance Company v. seescandy.com \& others, No C-99-0745 DLJ. 
terceros que utilicen en el tráfico económico, sin su consentimiento, una marca o signo idéntico o semejante para distinguir productos o servicios idénticos o similares, cuando la semejanza entre los signos y la similitud entre los productos puedan inducir a errores".

En otras palabras, en caso que un sitio en la Red intente confundir a los usuarios de Internet respecto a la titularidad o patrocinio del mismo por parte de un dueño de una marca registrada, este podrá accionar en su contra amparándose en la acción recién expuesta.

Amén de la tutela jurisdiccional, le queda el camino de dirigirse a los Centros de Arbitraje y Mediación reconocidos por ICANN ${ }^{3}$, a través de los cuales la parte afectada puede demandar en línea al infractor y conseguir a través de un procedimiento ágil y sumamente económico que el dominio sea desconectado ${ }^{4}$.

De esta manera vemos que el problema del uso de metatags como medio de facilitar la confusión con marcas registradas es fácilmente soluble a través del derecho vigente, sin plantear problemas jurídicos nuevos que merezcan un análisis ulterior.

3.2. Uso de metatags sin riesgo de confusión con una marca Registrada. A pesar de lo expuesto, quedan, sin embargo, en pie importantes dudas que trataremos en los acápites siguientes en profundidad. En primer término está el problema del riesgo de confusión fuera de la Ley de Marcas, es decir, cuando una empresa se intenta hacer pasar por su competidor sin usar marcas registradas que le pertenezcan. Otro problema diferente es el uso de metatags cuando no se deriva de ellos riesgo de confusión alguno, sea que se usen o no marcas registradas de un tercero, como si por ejemplo se incluyese entre los metatags de Coca-Cola el nombre Pepsi, identificándose claramente la página como perteneciente a su competidor, y finalmente la dilucidación de las hipótesis de uso legítimo de los mismos.

Dividiremos la materia en secciones diferentes según el problema a tratar.

3.2.1. Riesgo de confusión fuera de la Ley de Marcas. Se trata de un supuesto que no se ha presentado propiamente en el medio norteamericano, por lo que deberemos prescindir de la jurisprudencia de tal país para su análisis ${ }^{5}$. Consiste en que una empresa, a través del uso de nombres de dominio y metatags, intente captarse la clientela de su competidor a través de actos que generen la falsa creencia en el público consumidor de que se encuentra asociada o está relacionada de alguna manera con ella, pero sin que se encuentren en juego intereses protegidos por marcas registradas.

\footnotetext{
${ }^{3}$ ICANN es una organización sin fines de lucro que tiene por finalidad otorgar los dominios de Internet a las personas que los soliciten. Ella misma asigna los dominios de primer nivel, tales como .com, .org, .edu y otros similares. Asimismo, tiene un delegado en cada país que otorga los dominios de segundo nivel (.es, .ar, .cl, por ejemplo), los que se rigen por su normativa internacional para tal efecto.

${ }^{4}$ Para más detalles sobre este interesante procedimiento véase la normativa de ICANN.

5 Si bien es cierto que en el conocido caso Oppedabl \& Larson Advanced V. Concert se planteó una argumentación en esta línea, la verdad es que fue resuelto utilizando criterios diferentes a través del desarrollo del concepto de confusión inicial, que se encuentra más cercano a la competencia parasitaria que estudiaremos en el acápite siguiente.
} 
Un ejemplo sería una firma de arquitectos que confecciona su página en la Red usando en sus metatags el nombre de su competidora directa y agregando dentro de la página referencias suficientes como para que el usuario creyese erróneamente que se trata de la misma empresa o de una filial de ella. De esta manera el engaño se realizaría a través de dos etapas, una referida a los metatags para captar la atención del cliente, y una segunda para perfeccionar el engaño a través de indicaciones en la página misma que indujesen a confusión.

En el caso en cuestión nos escapamos del ámbito del conflicto entre dominios y marcas, tomando los metatags un rol más distintivo y autónomo dentro de la configuración del problema jurídico. Si bien el papel de los metatags aparece aun ligado a otras conductas que induzcan a perfeccionar la confusión, y por lo tanto no es tampoco un caso "puro" de utilización de los mismos, es cierto que el asunto presenta una mayor autonomía que jurídica que el problema anterior.

En este tipo de cuestiones adquiere relevancia la Ley de Competencia Desleal, que configura una tipología propia de situaciones en que el derecho de una empresa a participar distintivamente en el mercado es minado por sus competidores de manera fraudulenta.

Especial importancia reviste el art. 6 de tal normativa que dispone:

"Se considera desleal todo comportamiento que resulte idóneo para crear confusión con la actividad, las prestaciones o el establecimiento ajenos.

El riesgo de asociación por parte de los consumidores respecto de la procedencia de la prestación es suficiente para fundamentar la deslealtad de una práctica”.

Como se observa a primera vista, se trata de una norma tecnológicamente neutra, en el sentido que no especifica los comportamientos sancionados en base a los medios para provocar la confusión, sino más bien prescindiendo de ellos apunta al resultado concreto que producen, a saber, "crear confusión con la actividad, las prestaciones o el establecimiento ajenos", como también "riesgo de asociación".

Como señala la más autorizada doctrina: "En este particular se condensa el criterio de desvalor de la práctica. En consecuencia, la deslealtad de la confusión no es consecuencia del aprovechamiento del buen nombre de otro para captar clientes, sino de la introducción en el proceso de comunicación con la clientela de elementos que son adecuados para provocar preferencias o decisiones de mercado... fundadas en una falsa o incorrecta representación de la realidad acerca de la identidad o procedencia empresarial o profesional de la actividad, prestaciones o establecimiento considerados"6. De esta manera, el supuesto de comunicación comprendido bien puede ser el empleo de metatags y páginas en la Red que tengan por efecto crear la confusión o el riesgo de asociación, puesto que aquí se "tipifica el acto de confusión genérico, cualesquiera que sean los medios empleados para generar la posible confusión y cualquiera que sea la esfera de la actividad económica en que se produzca"7.

${ }^{6}$ MASSAGUer (1999) p. 167.

7 MASSAGUER (199) p. 168. 
Rige el tipo del art. 6 de la LCD para aquellos casos en que no se encuentren en juego intereses protegidos por el Derecho de Marcas, puesto que la LCD no busca un simple reforzamiento jurídico de las sanciones por infracción de marcas, sino que más bien tiene por finalidad proteger situaciones diferentes no amparadas por tal derecho, como es el ejemplo propuesto.

Ante la situación propuesta, sería posible, por tanto, ejercer las acciones especificadas en el art. 18 de la LCD, entre las que se cuentan la cesación de la conducta desleal y la reparación de los perjuicios causados, por lo que podemos concluir que este tipo de casos se encuentra amparado por el Derecho español.

En fin, podemos señalar que al tratarse de una materia armonizada, la protección será efectiva en todo el ámbito de la Comunidad Europea, lo que refuerza la eficacia de la misma.

3.2.2. Casos de competencia parasitaria con uso de metatags. A diferencia de las dos situaciones anteriormente analizadas, el presente grupo de casos se distingue por la ausencia de la posibilidad de confusión para los consumidores por el mero uso de metatags.

El conflicto modelo para la presente situación nos lo proporciona el caso "Oppedahl \& Larson v. Advanced Concepts"8. En este la prestigiosa oficina de abogados norteamericana Oppedahl \& Larson demandó a Advanced Concepts por haber incluido en las páginas de Red de sus clientes (otros estudios jurídicos) repetidas veces en sus metatags el nombre de la actora. De esta manera, cada vez que un usuario de Internet introdujese en algún buscador el nombre de la firma, aparecerían junto a ella una larga lista de las páginas de sus competidoras.

El caso era difícil de argumentar de acuerdo a la legislación estadounidense, ya que en Texas, lugar donde se desarrolló el litigio, los nombres de los estudios jurídicos no tienen consideración de marcas. Por otra parte, aunque los buscadores de Internet, como Yahoo o Altavista, podían confundir las páginas de las firmas competidoras con la de la actora, esto no era necesariamente aplicable al público, pues dichos sitios de la Red no contenían afirmaciones que hiciesen creer al usuario que tales estudios estuviesen relacionados en manera alguna con Oppedahl \& Larson. Por otro lado, cualquier navegante de Internet sabe por experiencia que es muy usual que los resultados de las búsquedas realizadas incluyan numerosas páginas que no tienen conexión alguna con aquella requerida.

La demandante intentó demostrar que la sola inclusión de metatags invisibles para el usuario le haría creer que existía algún tipo de asociación entre los titulares de las páginas obtenidas en una búsqueda, situación obviamente irreal desde el punto de vista del consumidor medio.

Otro camino intentado fue el alegar que tal actividad ocasionaba una dilución de la marca "Oppedahl \& Larson", ya que al generalizar su nombre para búsquedas de

\footnotetext{
${ }^{8}$ Civ. No 97-Z-1592 (D.C. Colo. complaint filed July 23, 1997).
} 
firmas de abogados, perdía su poder identificatorio y distintivo de un estudio determinado. Tal línea argumentativa muestra también una seria debilidad, puesto que al no considerarse el nombre de una firma de abogados como una marca, difícilmente se le puede atribuir un poder identificatorio tan elevado como para verse afectada por la dilución. Por otro lado, para los usuarios ni siquiera era visible el metatag que contenía dicho nombre, por lo que sería difícil creer que tal conducta afectara su poder individualizador.

Finalmente, el conflicto fue resuelto a través de un interesante concepto conocido como confusión inicial, el cual fue progresivamente desarrollado por la jurisprudencia norteamericana, encontrándose muy bien expuesto en la sentencia del caso "Brookfield Comunications, Inc. v. West Coast Entretainment Corporation”.

El razonamiento de la sentenciadora consiste en considerar que los metatags producen una confusión inicial en los usuarios, la cual después es desmentida por la página misma, pero que no obstante tiene relevancia jurídica.

De esta manera, al encontrar diferentes páginas en los resultados de la búsqueda, el consumidor no sabe exactamente cuál de ellos corresponde efectivamente a la empresa que busca. Aunque con un somero análisis el usuario dará con la página que se proponía encontrar, y no obstante no haber riesgo de confusión final, puesto que las páginas se diferencian lo suficiente como para que sepa en qué tienda compra, lo cierto es que ha logrado acceder a las páginas de los competidores de la compañía en cuestión a través del uso del nombre de la misma. En otras palabras, las empresas competidoras, en su afán de acceder al mercado, se están "colgando" del buen nombre de la empresa dominante. Consecuentemente, el esfuerzo realizado en publicidad, en mercadeo y, en general, en la obtención de una reputación por parte de la empresa afectada, redunda en beneficio de sus competidores.

De ahí proviene el origen del reproche de deslealtad que la jurisprudencia norteamericana asigna a tal conducta, puesto que en base a la reputación y buen nombre de una empresa sus competidoras obtienen indudables beneficios, ahorrándose ellas los costos necesarios para la formación del mismo.

En todo caso, la cuestión fue decididamente separada de los actos de comparación, que analizaremos más adelante, pero puede afirmarse sin vacilar que este uso de metatags es considerado ilícito en el Derecho estadounidense.

La situación en España es difícil de encuadrar dentro de los supuestos tradicionales de la competencia desleal.

Aparentemente, el tipo adecuado es aquel señalado en el art. 12 de la LCD que establece:

"Se considera desleal el aprovechamiento indebido, en beneficio propio o ajeno, de las ventajas de la reputación industrial, comercial o profesional adquirida en el mercado. En particular, se reputa desleal el empleo de signos distintivos ajenos o de denominaciones de origen falsas acompañados de la indicación acerca de la verdadera procedencia del producto o de expresiones tales como 'modelo', 'sistema', 'tipo', 'clase' y similares". 
El tipo en cuestión se centra en la explotación de la reputación ajena. Se ha señalado que "su ilicitud se funda en el recurso a la fama o reputación ajenas para presentar la propia oferta en el mercado y atraer así a la clientela" ${ }^{\text {, }}$ lo que parece compatible con la posición adoptada por la jurisprudencia norteamericana.

En el tipo en cuestión, el centro de la acción desleal lo constituye el expolio del esfuerzo ajeno en construir una reputación en el mercado, beneficiándose con ello sus competidores.

El problema es determinar si el uso de los signos distintivos ajenos, como una denominación de origen, su nombre comercial u otros en los metatags basta para ser considerado como un uso desleal de los mismos.

En nuestra opinión lo es, ya que cada vez que se efectúe una búsqueda asociada al signo distintivo de una empresa, aparecerán también junto a ella todos sus competidores, quienes no han realizado esfuerzo alguno a fin de obtener una reputación propia, sino que simplemente se adhieren injustificadamente a la de aquel. Parece que constituye un verdadero aprovechamiento indebido de tal reputación, puesto que los competidores, sin contribuir en nada al desarrollo de tal reputación, obtienen, no obstante, un beneficio de ella que va en directo desmedro de la posición comercial del afectado. Aunque el consumidor no sepa que dicho beneficio se produce, puesto que no tiene conocimiento de la inclusión de los metatags que son invisibles, es evidente que a través de los signos distintivos de una empresa llega a tomar conocimiento de las de sus competidores, quienes se enriquecen en base a la reputación de aquel.

Evidentemente, no basta que los signos distintivos de una empresa se encuentren incluidos en los metatags de otra para calificar de desleal la conducta, puesto que deben además concurrir los demás presupuestos propios de tal tipo, como son (1) la reputación del signo, (2) su implantación en el mercado, (3) el esfuerzo realizado para propiciar su reputación, (4) la proximidad competitiva y (5) lo indebido del aprovechamiento ${ }^{10}$, pero todas estas circunstancias deben determinarse caso a caso y su análisis pertenece al Derecho de la Competencia más que al del Comercio Electrónico.

3.3. Casos de uso legítimo. Una vez concluido el estudio de los casos de ilicitud más saliente en la utilización de los metatags, nos corresponde hacer una breve reseña de los supuestos de utilización legítima en los metatags de la marca o de los signos distintivos ajenos. Dividiremos su estudio en tres supuestos básicos, la calificación profesional, la oferta de productos complementarios y los actos de comparación, aunque no excluimos la posibilidad de que existan otras situaciones en que su uso sea justificado.

3.3.1. Calificación profesional. El caso que sentó jurisprudencia a este respecto lo constituye "Playboy Enterprises v. Welles"11. En él PEI demandó a la modelo Terri Welles por el uso de las marcas Playboy y Playmate en los metatags de su página en la Red.

\footnotetext{
${ }^{9}$ Massaguer (1999) p. 364.

${ }^{10}$ Información detallada se encontrará en el mismo MASSAGUER (1999) pp. 368 y ss.

${ }^{11}$ No 98-CV-0413-K JFS (SD CA, 22 de abril, 1998).
} 
El punto es que Terri Welles adquirió fama a principios de los años 80 tras aparecer en la revista Playboy en diciembre de 1980, siendo posteriormente elegida como Playmate del año en 1981. De esta manera, al diseñar una página para sus admiradores incluyó tales marcas dentro de sus metatags, aclarando en el hipertexto de su página que no estaba auspiciada ni vinculada en forma alguna a PEI.

La demanda fue rechazada por la sentenciadora argumentándose que Terri Welles simplemente usaba la referencia como medio de describirse a sí misma de una manera apropiada en su vida profesional, sin buscar confundir de manera alguna al público respecto a la procedencia de la misma.

De hecho, el punto es que sería difícil para una modelo el dar una idea adecuada de su carrera sin hacer mención de los títulos que ha obtenido en razón de su belleza. El otorgamiento de tales distinciones la habilita para emplearlas como un medio justo de describir su calificación profesional, pues establecer una doctrina contraria sería obligarla a pagar derechos de marca cada vez que intentase presentar su curriculum vitae.

Otros casos imaginables y analogables se podrían producir respecto a los deportistas. La única manera de que un jugador de fútbol describa su carrera es mencionando los equipos en que ha participado, por lo que es lógico incluirlos dentro de los metatags de su página en la Red, no obstante que correspondan a marcas registradas.

En definitiva, podemos decir que existe una autorización tácita para emplear los signos distintivos de una empresa para los empleados o ex empleados de ella, toda vez que lo hagan a efectos de describir su carrera y trayectoria, no obstante que representen una competencia para la misma, puesto que su aprovechamiento está justificado por los servicios que le prestaron en su oportunidad a aquella empresa y por la necesidad de establecer los méritos de su actuación y calificación.

3.3.2. Oferta de productos complementarios. Como es lógico, el Derecho de Marcas mismo impone ciertas limitaciones a la facultad de prohibir el uso de una marca registrada a terceros, las que son también aplicables a su utilización en metatags.

Un ejemplo tradicional lo constituye el caso de quien vende repuestos para una determinada marca de automóviles, no obstante no ser un distribuidor autorizado de los mismos. Lógicamente, la única manera de individualizar tales repuestos es a través de la identificación de la marca y modelo del automóvil al que van destinados, lo cual es lícito, siempre que no induzca a confusión respecto a su origen ${ }^{12}$. Lo dicho puede aplicarse a otros casos de similar naturaleza.

Un caso más problemático es aquel en que una empresa utiliza el nombre de otra para ofrecer productos que suelen acompañar a los de ésta. El ejemplo más claro lo constituye el de una agencia de viajes que incluya el nombre de "Disney" entre sus metatags, a fin de ofrecer viajes a las personas que consulten sobre información relativa a aquel lugar.

12 Se puede encontrar una exposición sucinta y clara en CHUlí́ (2000), p. 598. Para un estudio más detallado es recomendable leer STJCE, 23-2-1999, BMW c. Ronald Karel Deenik, Act. Trib., 5/99. 
En la jurisprudencia norteamericana no se ofrecen ejemplos que diluciden la cuestión, pero intentaremos igualmente expresar algo al respecto. Si bien la agencia de viajes se está beneficiando del buen nombre de Disney para obtener beneficios sin haber colaborado a su establecimiento, lo cierto es que el caso escapa de la competencia parasitaria, ello debido a que no existe una proximidad competitiva entre ambas empresas. Si Disney ofrece un parque de diversiones como producto, el ofertar viajes al mismo, lejos de mermar la posición competitiva de Disney, la mejora. En efecto, mientras más agencias de viajes ofrezcan sus servicios para conducir turistas a tal lugar, más popular se convertirá como destino, por lo que los beneficios que Disney obtiene se ven incrementados por la presencia de tales elementos. Podemos asociar la situación a aquella que se produce en el aparato digestivo de los seres humanos, donde conviven una multitud de parásitos que se benefician de los alimentos ingeridos por un hombre, pero al mismo tiempo, mediante su actividad, simplifican la digestión y favorecen el funcionamiento regular del cuerpo. La relación es, en alguna medida, simbiótica, pues otorga un beneficio mutuo y recíproco tanto a Disney como a las agencias de viajes, lo mismo que el hombre y sus parásitos digestivos.

Lo antes dicho es válido siempre que en la página respectiva se aclare debidamente que no existe asociación entre el dueño de la marca o signo distintivo y la empresa que usa el metatag, pues de lo contrario se caería en los tipos respectivos de confusión, sea de la Ley de Marcas, sea de la Ley de Competencia Desleal, según si el signo distintivo es o no una marca.

3.3.3. Actos de Comparación. Finalmente debemos señalar que tanto la jurisprudencia norteamericana como la LCD reconocen el derecho de los competidores de realizar actos de comparación de sus productos.

En el medio español, tal situación está reconocida específicamente por el art. 10 de la LCD, que admite como válida una comparación con tal que se sujete a ciertos requisitos, a saber, que se refiera a extremos análogos, relevantes y comparables.

No entraremos a especificar el detalle de la disposición, puesto que ello escapa a las necesidades de la presente exposición y pertenece, más bien, al Derecho de la Competencia, pero nos contentaremos con señalar que es posible el uso de metatags con el signo distintivo de un competidor a fin de realizar un acto de comparación, siempre y cuando tal actividad no rebase los límites propios de la misma y caiga en un caso de competencia parasitaria ${ }^{13}$.

\section{BIBLIOGRAFÍA CITADA}

CHUliá, Francisco Vincent (2000): Introducción al Derecho Mercantil (Ed. Tirant lo Blanch, Valencia), 886 páginas.

13 Nuevamente hemos de remitir al lector que desee profundizar en el tema a MASSAGUER (1999), pp. 309 y ss. 
ICANN, Normativa, [consulta 15 de julio de 2006] disponible en http:// www.activas.net/V1/nosotros/reglasICANN.php

JACKSON, Tim, Financial Times, 22/9/1997 [fecha de consulta 12 de julio de 2006] disponible en http://cyber.law.harvard.edu/metaschool/fisher/linking/meta/ meta6.html

Massaguer, José, Comentario a la Ley de Competencia Desleal, Civitas Ediciones, 1999, Madrid, 652 páginas.

\section{NORMAS CITADAS}

Lanham (Trademark) Act de 1946, Public Law 79-489, Título 15, capítulo 22 del United States Code.

Orden de 21 de marzo de 2000 del Ministerio de Ciencia y Tecnología, publicada en el Boletín Oficial Español (BOE) el 30 de marzo de 2000.

Ley 3/1991 de 10 de enero de 1991, de Competencia Desleal, BOE 11 de enero de 1991.

Ley 17/2001 de 7 de diciembre de 2001 de Marcas, BOE 18 de diciembre de 2001

\section{JURISPRUDENCIA CITADA}

"Columbia Insurance Company v. seescandy.com \& others", No C-99-0745 DLJ [fecha de consulta 18 de julio de 2006] disponible en http://www-personal.umich.edu/ -omri/ColumbiaInsurance_v_SeeCandy.pdf

"Oppedahl \& Larson v. Advanced Concepts" Civ. No 97-Z-1592 (D.C. Colo. complaint filed July 23, 1997) [fecha de consulta 10 de julio de 2006] disponible en http:// www.oppedahl.com/ac/complain.htm

"Playboy Enterprises v. Welles", No 98-CV-0413-K JFS (SD CA, 22 de abril, 1998) [fecha de consulta 20 de julio de 2006] disponible en http://www.loundy.com/CASES/Playboy_v_Wells.html

STJCE, 23-2-1999, BMW c. Ronald Karel Deenik, Act. Trib., 5/99 [fecha de consulta 22 de julio de 2006] disponible en http://europa.eu.int/eur-lex/es/archive/1999/ c_12119990501es.html 
